\title{
Computer Network safety and Precautionary Measures in the Big Data Era
}

\author{
Zou Nu, Liu Xiaoyan \\ Jiangxi Tellhow Animation Vocational College, Nanchang, Jiangxi, 330200
}

Keywords: real-time big data, processing technology, state monitoring, filed, application

\begin{abstract}
With the increasing of information technology in China, and the intelligence of network building, the requirements for safety detection technology of network operation are raising both in depth and width. The state detection work needs to gather a large amount of data information, and the operation of network system involves many important factors. Only by conducting all-around control can the operation, production and management in all industries are driven. It is suggested that, the application advantages of safety detection system should be taken to demonstrate the value in applying technology. Particularly in the big data era, the application of computer network safety and precaution processing technology often manifest in the system monitoring. If it fails, the utility of information technology will be hard to be guaranteed, and the operation safety cannot be improved. Therefore, the authors believed that, it is necessary to have a comprehensive knowledge about the concept of computer network safety, and summarize and divide problems in the network safety. Then, the authors proposed related theories to deal with the problems, which have formed a systematic precaution system, laying a solid foundation for the safe network operation.
\end{abstract}

With the acceleration of scientific and technological development in the recent years, people have realized the importance of safe operation and strengthened the cognitive ability in the application of network information system. Especially as the increasing of the number of data processing and monitoring in the storage state, the computer network safety as well as its precaution work has become an integral part in promoting the development of information technology. The authors hold that, monitoring related data information by advanced technologies and other technological means like state monitoring can manifest the comprehensive equipment operation and real features, which can drive the development of information technology, reduce errors of the safety failure, and discover hidden problems when the equipment is running.

\section{A brief analysis on the problems in big data and computer network safety}

\subsection{The concept of the bid data}

Broadly speaking, the so-called big data means storing huge data in a safe and comprehensive manner. The big data includes valuable information, which demonstrates the diversification of data information. And the diagram of big data running is shown in figure 1 . With progress of the time and the acceleration of the data processing rate by the computer, the cloud-computing is a core common method. Thus, it has changed the way of information and resource acquisition, changed the traditional servers, wired and wireless network into intangible terminal, which definitely indicating the advance of the time. The application of the big data boasts low value density, the large number, and various types with fast processing rate, thus, being widely used in all information sectors. However, the problems in the information transmission of computer network lead people to realize the significance of the information safety, in particular the rising of network crimes in the past few years. Therefore, it is necessary to strengthen precaution work in information safety and create a sound running environment. [1] 


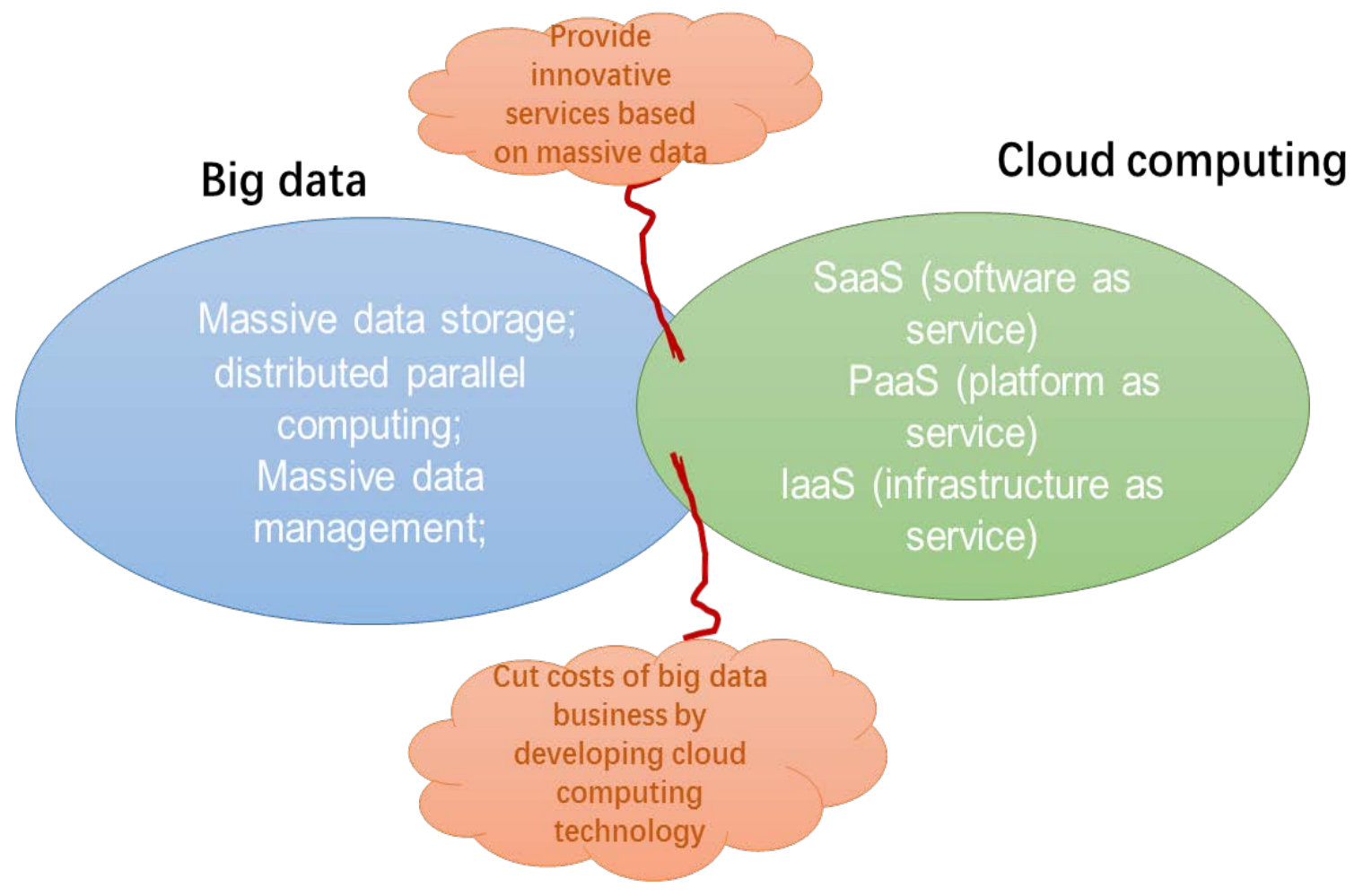

Figure 1. diagram of the big data running

\subsection{Potential threats in running computer network}

The computer network security refers to the application of network technology or management mechanism under a relatively safe environment to ensure the integrity, impermeability, and practicability of the computer network information. Against the big data era, the rich network medium, various information transmission methods have brought about more and more factors impacting the network safety including system bug, human factor, constituting the potential threats in the network information transmission. [2]

\section{The summary of the problems in computer network safety in the big data era}

\subsection{Problems in information safety}

Under the data era, the computer network consists of massive data information with rich contents. However, in the relatively open network environment, the safety of data transmission is hard to be guaranteed, and the practicability of data will decrease. The latent threats mainly include data destruction and information leakage. The authors, therefore, analyze the causes of these problems: virus attacks, theft or illegal interception by outlaw, which will destroy data information, bringing damages and threats on the economic interests of enterprises and individuals. [3]

\subsection{The safety problems caused by the network bug}

Theoretically, all bugs and problems will emerge in the computer network operation. Whether the Linux system or the Windows system is difficult to prevent the safety threats of the system itself, which may come from internal software or the restrictions in the hardware. Particularly in the installment of software, the bugs are easily ignored, laying hidden troubles. In most cases, the bugs brought by the system itself can be guarded against or controlled. However, the bugs and destruction made in software installment or downloading by users are hard to be prevented. Lawbreakers will steal user information by means of the software bug, bringing about latent dangers and problems. [4]

\subsection{Safety problems caused by misoperation}

The computer operation is easily influenced by human factors, posing threats on the computer 
network safety, which can be divided into hostile attacks and unintentional attacks. When the information technology is widely used, the operant level of users varies, and not all users can gasp all operation skills, and part of them misoperate because of the incomplete understanding for network safety rules, causing safety bugs of computers, offering opportunities for lawbreakers and hackers, and huge economic loss for users. Nonetheless, the safety problems in computer network caused by human factors are the major problems in network safety. The intentional and malicious destruction of a computer through decoding or interception by professional technologies will reveal personal confidential information, bringing great damage for the application of computer network technology. [5]

\subsection{Safety problems caused by poor management}

The computer network management can enhance the capacity of the preventing the outside invasion and create a safe operating environment. The authors believed the network management is a necessary work, and the following is the major safety problems caused by poor management.

First of all, the computer users will pay less attention to the routine maintenance due to poor management, bringing damages on the computer network system in a varying degree. [7]

Secondly, institutions and organizations like schools, banks and governments will implement systematic management in using computer network to avoid malicious attacks. However, loose management will hinder the operation of institutions and bring irreparable losses.

\section{The discussion of precaution methods of computer network safety in the big data era}

\subsection{The application of security detection system and firewall}

Given the common problems in safety of computer network, the building of systematic information security management mechanism can solve the problems timely. And the strengthening of management of network safety to prevent malicious attacks as well as virus threats are important technological means. [9] While firewall technology is used to enhance the safety of computer network by virtue of isolation of the topological structure.

Currently, the firewall technology is widely applied in enterprises and public network, which strengthens the security management, combine external management and internal management, and create a safe operating environment. People usually input data into the internal management mechanism, build systematic management mechanism internally and externally by the firewall to stifle security risks in the cradle, and avoid virus attacks.

As the increasing of the type of computer virus, the network security managers should notice the significance of implementing the precaution work against virus, and make a summarization of the principles and features of virus. Based on this, they should employ all-around virus detection, purchase advanced virus detection technologies, putting virus prevention into practice. For instance, antivirus program, password security, $U$ disk management software shall be regarded as important channels to ensure efficient security management and a sound computer operating environment. [10-12]

\subsection{Strengthening the network security management}

The managers of computers shall shoulder their responsibilities to prevent network bugs for lawbreakers. The authors, by analysis and comparison, learned that, any organizations or individuals should do a good job in management and routine maintenance in applying computer technologies, focus on network security, understand the integrity and precision of data information in the big data era, implement comprehensive data security management, take technological management as a guarantee, put related safety precaution measures into place so as to achieve rational, scientific and proper safety management.

In utilizing computer network, organizations and institutions should analyze from a macro perspective, construct a dynamic, ordered management mechanism, take network security precaution as a strong support to improve the safety and reliability of network system and eliminate 
all internal risks in the network operation.

In addition, technicians should realize the dangers brought by illegal links or phishing websites, ensure the preciseness of operation, prevent the accidents by misoperation.

\subsection{The application and installment of antivirus software}

The computer virus emerges following the increasingly enhancing computer network technologies in the big data era, bringing great threats on the operation of information management system. And common hostile virus in the computer system includes spyware, worm, and Trojan virus, thus, in applying computer technologies, many computer safety manufacturers have attached much importance on the research and development of cloud-security technologies. For example, Kingsoft Antivirus developed by Kingsoft is a typical case. As shown in figure 2, the cloud-security technology has created a sound operating environment for the computer network system to prevent invasion and interference of malware. What is more, lightweight defense software or safety detection software can be installed on the insulated computers, and safety detection engine can be applied in the cloud. All protective software can ensure a safe operating environment of computers, improved the reliability and stability of the computer system. [13-14]

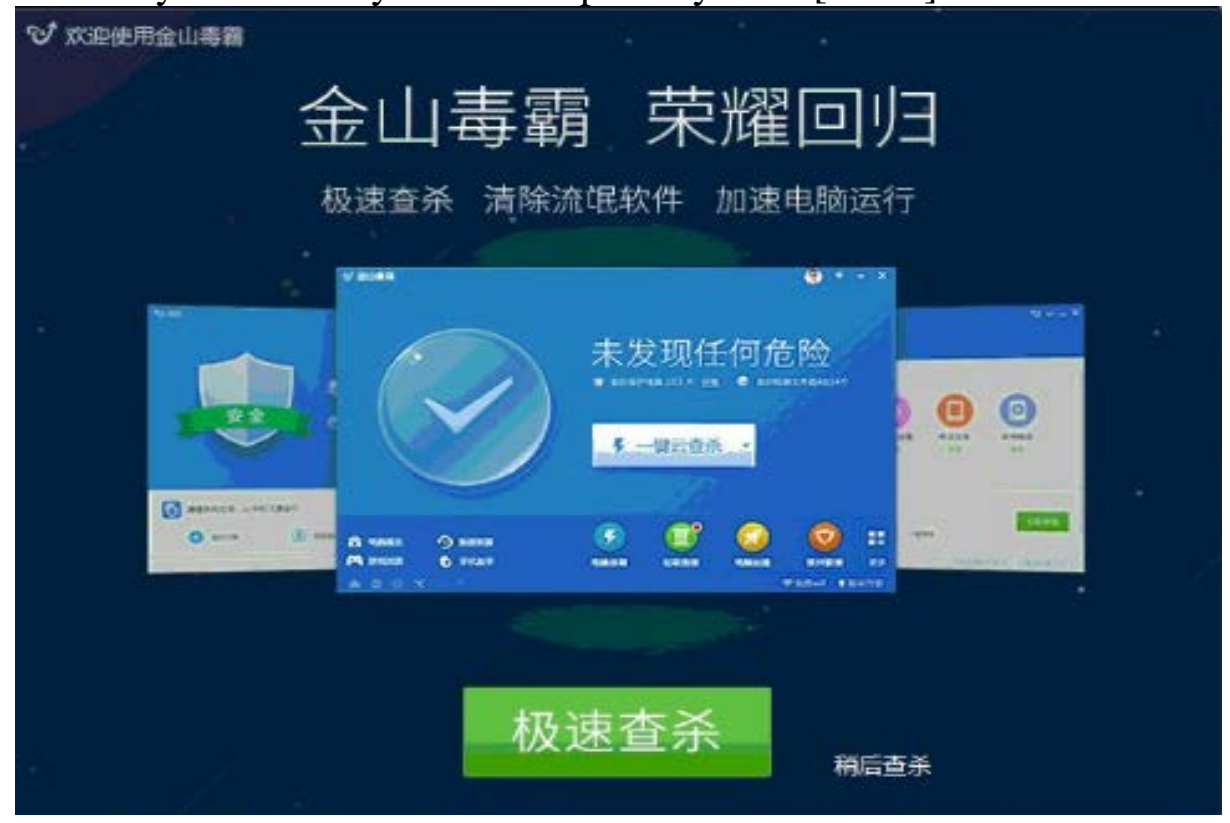

Figure 2 Kingsoft antivirus

\subsection{Strengthening security of information transmission and storage}

In order to ensure the transmission safety and the efficiency against the data era, it is inevitable to start from the information transmission and storage, and guarantee the precision and practicability of information data. The authors believe that, the information technology in the computer network can be taken to do the encryption work, protect the data transmission and storage, gather and integrate the information in the computer network, share and transmit messages in the form of ciphertext. In this way, various safety risks can be avoided, data theft and interception can be prevented. Even if the data information is stolen, the specific content is hard to know without the knowledge of ciphertext. Therefore, the security in transmitting and storing data information can be improved, and a sound data transmission environment can be created.

\section{Conclusion}

In a word, the information technology still develops, which requires research and discussion. Only in this way, can its development inject impetus into the growth of all walks of life, only in this way, can the computer technology be put into practice to ensure the security. The authors think that, it is necessary to make a summary of the problems in operating network, analyze and summarize the specific causes, and make the effect on life and production clear in this process. The security in 
running computer network plays a crucial role in all industries, whose stability and safety hold the key. Thus, the application of computer network should be considered from a global perspective with advanced monitoring technologies as the support, and failure should be coped with timely to ensure the integrity and efficiency of the data information storage inside the network.

\section{Acknowledgements}

Fund Item: The paper is supported by the science and technology project of Ministry of Education of Jiangxi Province, named Research and Application of Player Sentiment Analysis in the Online Games Based on Big Data (Item Number: GJJ171408).

\section{References}

[1] Zhang Shaomin, Mao Dong, Wang Baoyi et al. Application of big data processing technology in fault diagnosis and early warning of wind turbine gearbox [J]. Power system automation,2016,40(14):129-134.

[2] Zhu Haojun, Liang Zhenfen. Case analysis of the state monitoring of low-speed paper equipment [J]. Equipment management and maintenance,2015,15(5):73-77.

[3] Zhou Guoliang, Zhu Yongli, Wang Guilan et al. Application of real-time big data processing technology in the field of state monitoring [C]. // academic BBS proceedings of the 6th electrical and technological frontier issues.2014:432-437.

[4] Qiao Xinjian. Research progress of wireless sensor network for monitoring the status of mechanical equipment [J]. Commodity and quality,2015,26(8):74.

[5] Yang Pinfu, Wan Tao, Chen Xianqiao et al. Design and application of the remote monitoring system of the channel surveying ship [J]. Journal of wuhan university of technology (information and management engineering edition),2013,35(5):643-645,649.

[6] Bi Runzhen. Reflections on the maintenance and state monitoring of relay protection [J]. Scientific Chinese,2014,15(23):140-140.

[7] Liu Lixia. Research on adult teaching quality in the context of big data [J]. Continue education,2017,29(5):15-18.

[8] Li Jingbo. Application of large data technology in monitoring and evaluation of operation state of wind power units [J]. Electromechanical information, 2016,16(9):67-68.

[9] Ji Xiaofeng. Application of big data in the key mobile device field of cnooc offshore oil platform [J]. Technology and innovation, 2011,11(16):149-151.

[10] Wang Wenjie. The monitoring system of computer room environment based on iot technology [J]. Guangdong science and technology,2014,11(20):44-45.

[11] Zhang Kun, Zhang Tiemin, Liao Yiyong, etc. Based on Labview, real-time monitoring and evaluation system design of uav flight status [J]. Journal of agricultural engineering,2016,32(18):183-189.

[12] Li Bo, Dong Zhenping, Yu Junqi et al. Application of big data technology in the durability monitoring system of industrial building structures [J]. Digital technology and application, 2011,22(4):106-107.

[13] Yang Xiaolai. Design of intelligent environmental data detection system based on ZigBee technology [J]. Communication world,2015,11(23):263-263.

[14] Guo Rongyu. Application of big data technology in the health monitoring of large span steel structures [J]. Digital user,2017,23(34):55-56. 